\title{
Assessment of the Correlation Between Mother and Child Body Mass Index and Mother and Child Diet in Children With Food Allergies
}

\author{
Yasuko Fukudaa, d, Makoto Kameda ${ }^{\text {b, c }}$
}

\begin{abstract}
Background: Children with food allergies (FA children) tend to be smaller in size, mothers of FA children (FA mothers) tend to have a significantly lower body mass index (BMI) than those of non-FA children (N mothers), and FA mothers' diets tend to be synchronous with the substitute diets for their children. The study aims to examine the correlation between the diets of FA children and FA mothers as well as the BMI of FA mothers.
\end{abstract}

Methods: In total, 41 pairs of FA children (age: $6.5 \pm 2.5$ years) and FA mothers (FA group) and 37 pairs of children without food allergies ( $\mathrm{N}$ children, age: $7.0 \pm 2.7$ years) and $\mathrm{N}$ mothers ( $\mathrm{N}$ group) were included. BMIs of mothers and children, nutrient intake adequacy, correlation of meal content between mothers and children, and sampling of the combined dietary patterns of mothers and children were compared and contrasted to identify factors that affected the BMIs of FA mothers.

Results: There were no differences in BMI between the two groups of children, confirming the typical growth of FA children. BMIs of FA mothers were significantly smaller than those of $\mathrm{N}$ mothers $(\mathrm{P}$ $=0.038$ ). The intake adequacy of sweets was significantly higher in FA mothers than in $\mathrm{N}$ mothers $(\mathrm{P}=0.041)$. The correlation of meal contents between mothers and children was significantly higher in the FA group than in the $\mathrm{N}$ group $(\mathrm{P}=0.019)$. However, there was a discrepancy in the combined dietary patterns (first principal component) between mothers and children in the FA group. There was a significant negative correlation between the BMIs of FA mothers and responses to the use of substitute food $(\mathrm{P}=0.016)$.

Manuscript submitted August 16, 2019, accepted September 13, 2019

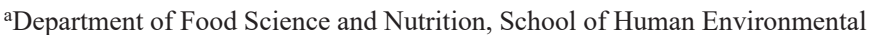
Sciences, Mukogawa Women's University, 6-46 Ikebiraki-cho, Nishinomiya, Hyogo 663-8558, Japan

${ }^{b}$ Department of Pediatrics, Osaka Prefecture Medical Center for Respiratory and Allergic Diseases, 3-7-1, Habikino, Habikino, Osaka 583-8588, Japan

'Osaka Habikino Medical Center, 3-7-1, Habikino, Habikino, Osaka 583-8588, Japan

${ }^{\mathrm{d} C o r r e s p o n d i n g ~ A u t h o r: ~ Y a s u k o ~ F u k u d a, ~ D e p a r t m e n t ~ o f ~ F o o d ~ S c i e n c e ~ a n d ~}$ Nutrition, School of Human Environmental Sciences, Mukogawa Women's University, 6-46 Ikebiraki-cho, Nishinomiya, Hyogo 663-8558, Japan.

Email: yafukuda@mukogawa-u.ac.jp

doi: https://doi.org/10.14740/jocmr3959
Conclusions: Low BMI in FA mothers may be related to substitute diets that differ from those of the FA children, consumption of sweets in dietary habits, and the use of substitute food.

Keywords: Body mass index; Children; Correlations; Food allergy; Mothers

\section{Introduction}

Body mass index (BMI) is one of the indicators for evaluating the degree of physical development during childhood $[1,2]$. Measurements of the percentages of body fat and body composition are essential in the evaluation of obesity and leanness [3]. However, because these measurements are not routine, BMI, which is conveniently calculated from height and weight and has been shown to be highly correlated with total fat [4, 5], is often used. BMI may serve as a comprehensive measure of health status and disease control in adults [6,7]. Children with food allergy (FA children) are reportedly small in size and often have low body weight [8,9], and the BMIs of their mothers (FA mothers) is reportedly significantly lower than those of mothers of children without FA (N mothers) [10].

Eating habits during early childhood are influenced by the caregiver's awareness and dietary habits [11]. In addition, the formation of lifestyle habits, including eating habits, during this period is considered important in reducing the risk of developing lifestyle-related diseases in the future [12]. Adults living in the same household have a significant impact on the nutrients and food groups that children consume [13]. Moreover, between both parents and their children, the mother and her child have higher similarities in their diet [14]. Additionally, if a child has a food allergy, the family's, especially the mother's, diet tends to be synchronous with the child's substitute diet [10].

Therefore, evaluating combined mother and child meal content and the body size and diets of FA children and FA mothers was considered meaningful. In this study, BMI at fixed points was calculated and compared for FA children and FA mothers and children without FA ( $\mathrm{N}$ children) and the mothers of non-FA children (N mothers) to assess physical build. In addition, the meal content of mothers and children was investigated and the individual findings for the mother and child, the correlation of meal content between mother and child and 
the degree of that correlation, and derivation and analysis of the dietary patterns of the mother and child combined were compared. Subsequently, the dietary factors that affected the BMI of FA mothers themselves were considered.

\section{Materials and Methods}

Two types of questionnaire surveys were conducted. The target population was 78 pairs of children and their mothers who visited the Department of Pediatrics at Osaka Prefectural Medical Center for Respiratory and Allergic Diseases (currently known as Osaka Habikino Medical Center) between July and August 2013. The breakdown was as follows: 41 pairs of FA children and FA mothers (FA group) and 37 pairs of $\mathrm{N}$ children and $\mathrm{N}$ mothers (N group).

Exclusion criteria included any physical or mental illness in the mother or a chronic illness other than allergies in the child or if the children and their mothers were deemed inappropriate for the study by the attending physician.

The purpose of this study was explained during the period when the mothers and children were waiting to undergo medical examinations in the outpatient ward, and responses to the questionnaires were requested from mothers who provided consent. For the $\mathrm{N}$ group, the investigator confirmed that the children did not have FA and there were no dietary restrictions due to other diseases.

\section{Profile survey}

The questionnaires asked for free responses on the age, height, and weight of the children and mothers. The child's height and weight values were obtained by checking the medical records on the day of consultation. The BMIs of the mother and child were calculated from this information. For FA children, the mothers were asked about the meal content causing the FA and the use or non-use of substitute food. BMI was calculated using body weight $(\mathrm{kg}) /$ height $(\mathrm{m})^{2}[15]$. The smoking status was not collected.

\section{Dietary survey}

The Food Frequency Questionnaire Based on Food Groups (hereinafter, FFQg) [16] was used for the children, whereas the Brief-Type Self-Administered Diet History Questionnaire (hereinafter, BDHQ) [17] was used for the mothers.

Nutrient intake was compared with the "Dietary Reference Intakes for Japanese 2015" [18] and the "Dietary Balance Guide: Ministry of Agriculture, Forestry and Fisheries" [19] and expressed as a percentage (\%) of the total intake, the target amount, and the recommended amount.

Ten food groups, excluding nonessential foods and seasoning spices, were used to evaluate the correlation of meal content between mothers and children. After calculating the correlation coefficient for each mother-child pair, the mean correlation coefficients for the FA and $\mathrm{N}$ groups were calcu- lated and compared between the two groups.

Dietary patterns combining the diets of the mother and child were analyzed by principal component analysis using 30 food groups, including nonessential foods and seasoning spices, in an attempt to derive factors affecting dietary patterns. Food groups that represented principal components were those with the load factors of $\geq 0.30$ and those that could be judged as "having a slight correlation." Dietary pattern analyses are widely used in a variety of ways [20].

Furthermore, the relationship between the BMI of FA mothers and the use of substitute foods was investigated using linear regression analysis with the mother's BMI as the objective variable and the child's age and sex, the mother's age, and the use of substitute foods as explanatory variables.

\section{Statistical processing}

SAS Version 9.4 (SAS Institute Inc., Cary, NC, USA) was used for statistical processing, and data are presented as mean \pm standard deviation (mean $\pm \mathrm{SD}$ ). The Welch $t$-test was used to compare differences in the average values between the two independent groups. Spearman's rank correlation analysis was used to evaluate the correlation of the meal contents of mothers and children. Principal component analysis was used to extract dietary pattern. Multiple regression analysis (stepwise) was used to test linear regressions. The statistical significance level was set to $\mathrm{P}<0.05$ in two-sided tests.

\section{Ethical compliance with human study}

This study was approved by the Ethical Review Board of the Osaka Prefectural Medical Center for Respiratory and Allergic Diseases (currently known as Osaka Habikino Medical Center) (Approval no. 633; approved on April 11, 2013) and the Research Ethics Committee of the Mukogawa Women's University and Junior College (Approval no. 13-06; approved on May 28, 2013), and was conducted in accordance with the Helsinki Declaration. The study content was explained to the subjects beforehand, and their written consent was obtained. Consent was also obtained for the use of sensitive information in this study, and personal information was pseudonymized when conducting the analysis.

\section{Results}

\section{Profile survey}

There were no differences between the BMIs of children in the FA and N groups. The BMIs of mothers were significantly lower in the FA group $\left(20.1 \pm 2.3 \mathrm{~kg} / \mathrm{m}^{2}\right)$ than those in the $\mathrm{N}$ group $\left(21.4 \pm 3.3 \mathrm{~kg} / \mathrm{m}^{2}\right)(\mathrm{P}=0.038)$ (Table 1$)$. There were significantly more alcohol consumers in the FA group than those in the $\mathrm{N}$ group ( $\mathrm{P}<0.001)$; however, the volume of alcohol consumption did not differ between the FA and $\mathrm{N}$ groups. Therefore, alcohol consumption was interpreted to have no major effects. 
Table 1. Demographics

\begin{tabular}{|c|c|c|c|}
\hline & Non-food allergy group & Food allergy group & P value \\
\hline $\mathrm{N}$ (pairs) & 37 & 41 & \\
\hline Age of children (years) & $6.9 \pm 2.7$ & $6.5 \pm 2.5$ & 0.107 \\
\hline BMI of children $\left(\mathrm{kg} / \mathrm{m}^{2}\right)$ & $16.0 \pm 1.9$ & $15.5 \pm 1.2$ & 0.207 \\
\hline Age of mothers (years) & $36.5 \pm 4.6$ & $37.1 \pm 5.4$ & 0.599 \\
\hline \multicolumn{4}{|l|}{ Alcohol consumption } \\
\hline Consumers/non-consumers & $14 / 13$ & $41 / 0$ & $<0.001 * * *$ \\
\hline $\mathrm{g} / 1,000 \mathrm{kcal}$ & $6.2 \pm 11.4$ & $6.6 \pm 4.0$ & 0.819 \\
\hline Total number of food causes: egg, milk, flour (piece) & - & $37 / 4$ & \\
\hline
\end{tabular}

Data are expressed as mean \pm standard deviation. Welch $t$-test. a Fisher's exact for proportion. ${ }^{*} \mathrm{P}<0.05,{ }^{* * *} \mathrm{P}<0.001$. BMI: body mass index.

\section{Dietary survey}

\section{Adequacy of nutrient intake}

There were no differences in the adequacy of energy-intake rate between the FA and $\mathrm{N}$ groups in both mothers and children.

In children, the nutrients for which the intake adequacy was significantly higher in the FA group than in the $\mathrm{N}$ group were vitamin $\mathrm{B} 1$, vitamin $\mathrm{C}$, potatoes, sugars, fruits (all $\mathrm{P}<$ $0.05)$, and meat $(P=0.001)$, whereas the intake adequacy of eggs was significantly lower in the FA group than in the $\mathrm{N}$ group $(\mathrm{P}<0.001)$.

In mothers, the nutrients for which the intake adequacy was significantly higher in the FA group than in the $\mathrm{N}$ group were protein, salt (both $\mathrm{P}<0.001$ ), vitamin $\mathrm{D}$, fishes, and sweets (all $\mathrm{P}<0.05$ ), whereas the intake adequacy for nonessential beverages was significantly lower in the FA group than in the $\mathrm{N}$ group $(\mathrm{P}=0.032)$ and tended to be lower for eggs $(\mathrm{P}$ $=0.074$ ) (Table 2). Sweets were grain-based confectioneries (e.g. rice confectioneries) that did not contain eggs or milk.

\section{Correlation of meal content between mothers and children}

The mean correlation coefficients were significantly higher in the FA group $(0.39 \pm 0.21)$ than in the $\mathrm{N}$ group $(0.29 \pm 0.18)$ (P $=0.019)($ Table 3$)$.

\section{Derivation of dietary patterns combining the diets of mother and child}

The principal component factors and load factors from groups 1 to 5 in the FA and $\mathrm{N}$ groups are shown in Table 4.
For the first principal component in the FA group, only elements related to the mothers' diets were extracted. The results were interpreted as "alternate mother diets" because significant results for pulses $(0.314)$, other vegetables $(0.315)$, and meat $(0.315)$ were derived in the positive direction in the mothers. For the second principal component, only elements related to the children's diets were derived. The results were interpreted as "non-substitute children's foods" because significant results for potatoes $(-0.331)$, other vegetables $(-0.352)$, meat $(-0.326)$, sweets $(-0.317)$, and seasoning spices $(-0.312)$ were extracted in the negative direction in the children.

For the first principal component in the $\mathrm{N}$ group, there were no load factors that were $\geq 0.30$, and for the second principal component, milk (0.355) was derived in the positive direction in children and fishes $(-0.302)$ in children and meat $(-0.355)$ in the mothers were derived in the negative direction. The first principal component was interpreted as "not meaningful," and the second principal component was interpreted as "preferences in the side dishes of the mother and child" (Table 4).

\section{Relationship between the BMI of FA mothers and the use of substitute foods}

There was a significant negative correlation between the BMIs of FA mothers and responses of "substitute food use" $(\beta=$ -7.651, $t$ value $=-2.465, \mathrm{P}=0.019$ ) (Table 5).

\section{Discussion}

We evaluated the BMIs and meal contents of FA children and FA mothers and investigated dietary factors affecting the BMIs of FA mothers. The strengths of this study are that a control group was set and the correlation of meal content between the mother and child and the dietary patterns for the combined 
Table 2. Adequacy of Nutritional Intake

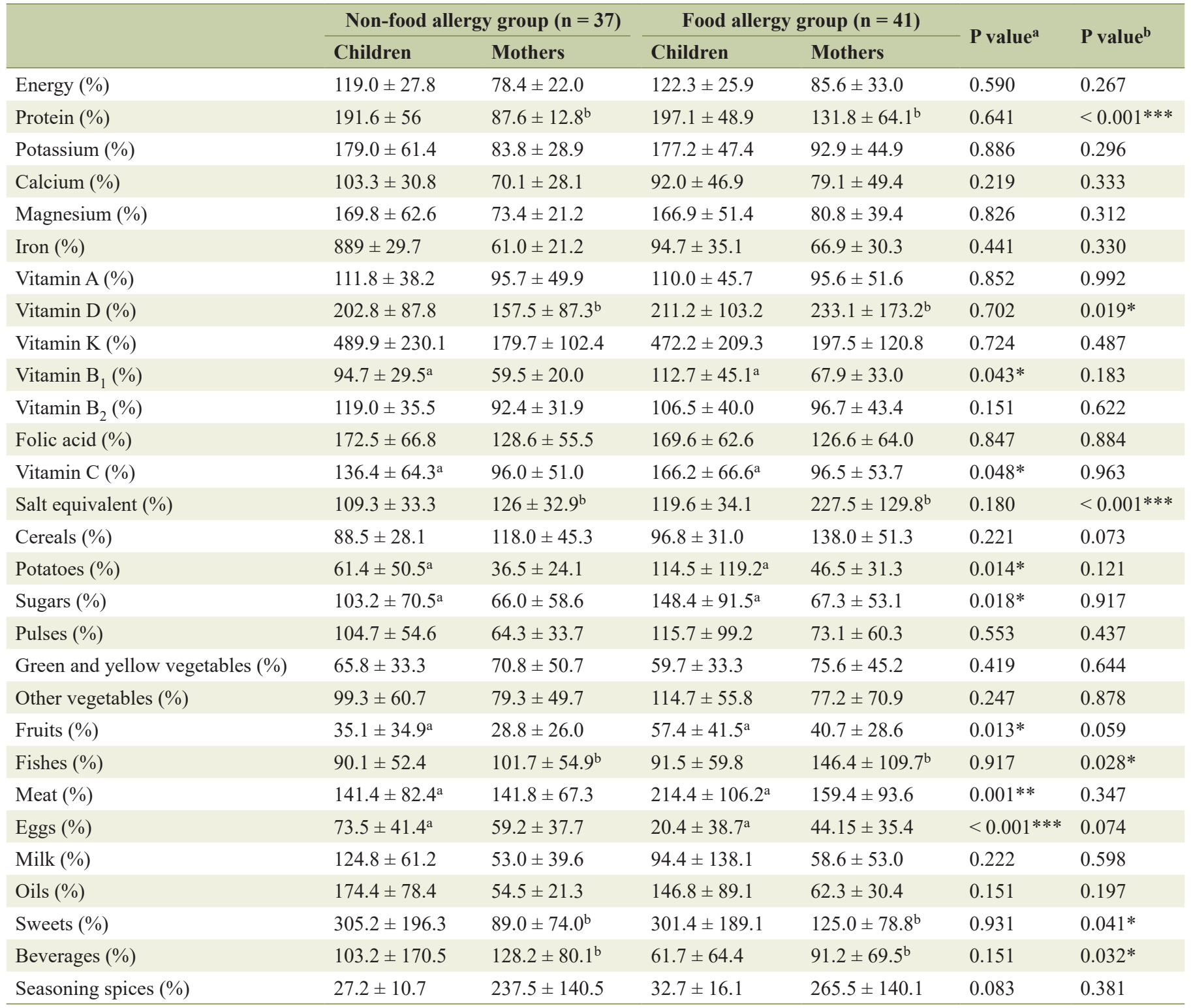

Data are expressed as mean \pm standard deviation. Welch $t$-test as appropriate. aP value: non-food allergy group children vs. food allergy group children. ${ }^{\text {b }} \mathrm{P}$ value: non-food allergy group mothers vs. food allergy group mothers. ${ }^{*} \mathrm{P}<0.05,{ }^{* *} \mathrm{P}<0.01,{ }^{* * *} \mathrm{P}<0.001$.

meal content of mother and child were derived, and these data were compared and evaluated.

In this study, there were no differences in the BMIs of children between the two groups. Meyer et al [8] and Chong et al [9] reported that FA children were smaller in size. However, our results indicate that there are no differences in BMIs between FA and N children, suggesting that FA children develop normally. This was thought to be because of the strict adherence to elimination-substitution diet therapy, resulting in the appropriate intake of nutrients necessary for the child's physi-

Table 3. Correlation of Meal Content Between Mother and Child

\begin{tabular}{llll}
\hline & Non-food allergy group $(\mathbf{n}=\mathbf{3 7})$ & Food allergy group $(\mathbf{n}=\mathbf{4 1})$ & P value \\
\hline$\rho$ & $0.29 \pm 0.18$ & $0.39 \pm 0.21$ & $0.019^{*}$
\end{tabular}

Data are expressed as mean \pm standard deviation. Spearman's rank correlation coefficient. ${ }^{*} P<0.05$. In the analysis method, after calculating the correlation coefficient for each mother-child pair, the mean correlation coefficients for the food allergy and non-food allergy groups were calculated and compared between the two groups. 


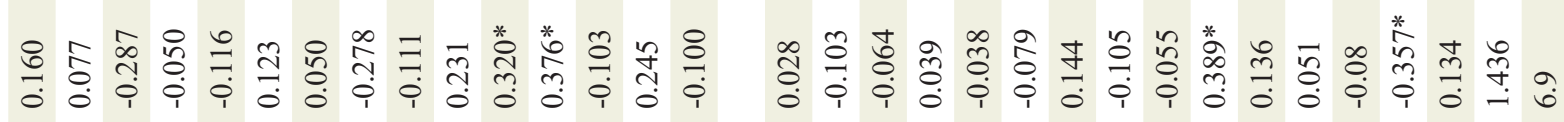

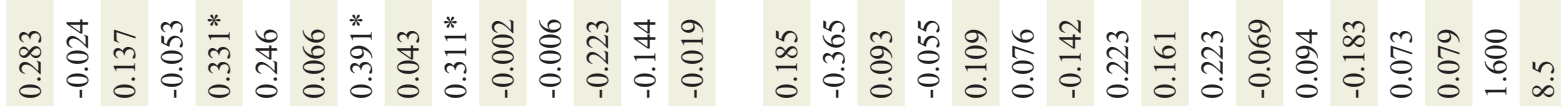

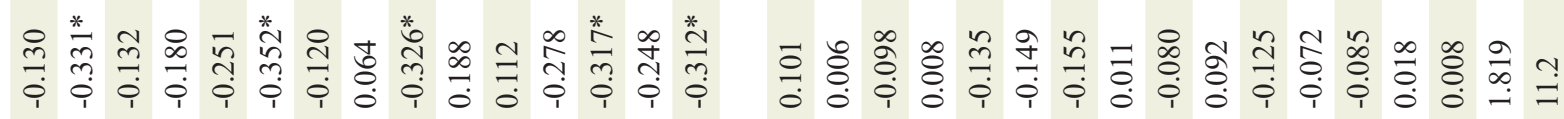

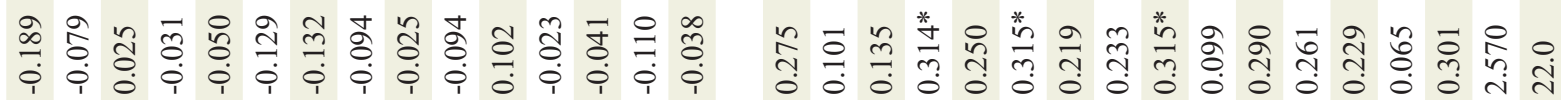

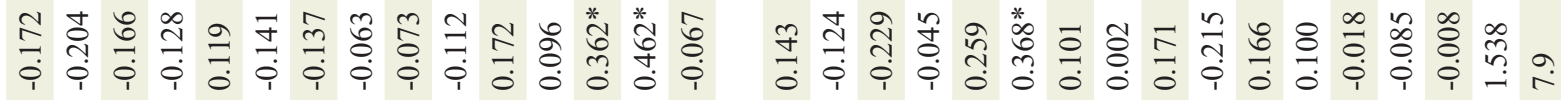

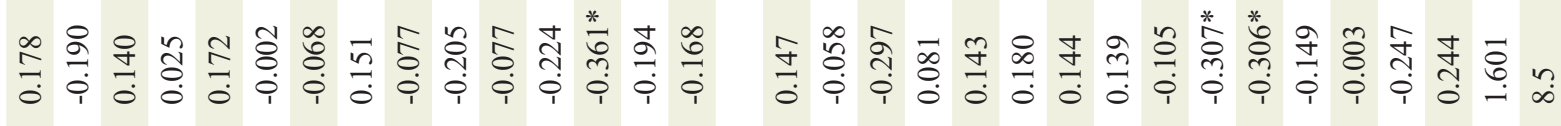

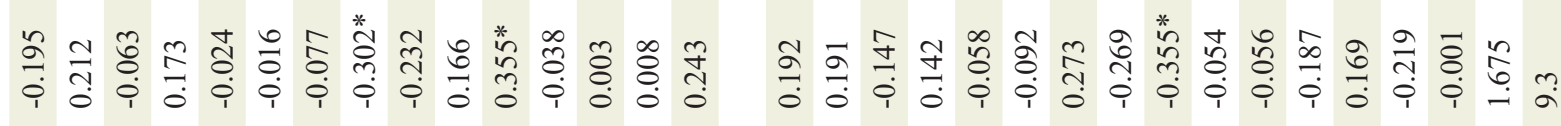

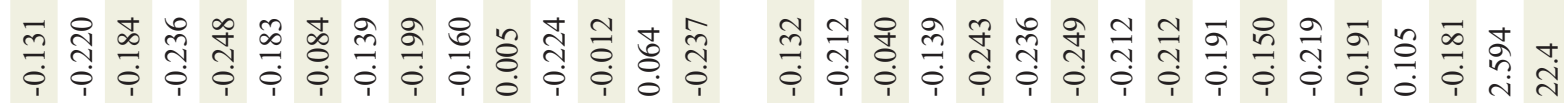


Table 5. Relationship Between the BMI of FA Mothers and the Use of Substitute Foods

\begin{tabular}{lllll}
\hline & Regression coefficient & Standard error & $t$ value & P value \\
\hline Substitute food use & -7.835 & 3.051 & -2.568 & $0.014 *$ \\
\hline
\end{tabular}

${ }^{*} \mathrm{P}<0.05$. Multiple regression analysis (stepwise). A linear regression analysis was performed considering the mother's BMI as the objective variable, and the child's age and sex, mother's age, and use of substitute foods as explanatory variables. BMI: body mass index; FA: food allergies.

cal growth.

In elimination-substitution diet therapy, foods with animal proteins, such as eggs and milk, that cause allergy are eliminated and protein is supplemented using substitute foods, such as meat, fishes, pulses, and other animal and plant protein foods, or substitute food is used to prepare the corresponding foods. The intake of appropriate quantities of energy and good protein is necessary for body growth, and a low BMI may be caused by a shortage of these nutrients in inappropriate substitute diets.

Proteins are important nutrients that make up our bodies (bone, muscle, viscera, blood, skin, etc.), enzymes, hormones, immune antibodies, etc. and comprise about 20 amino acids [21]. These include essential amino acids that cannot be synthesized in the body and must be consumed as food [22]. Proteins with balanced essential amino acids are regarded as high quality, and eggs, milk and dairy products, meat, fishes, and soybeans and soybean products are included as high-quality protein foods. The balance between animal and plant proteins is important in terms of amino acid balance and digestibility [18]. In FA children, both protein quality and quantity seemed to be appropriately supplemented.

However, there were differences in the BMIs of the mothers. To date, it has been reported that the BMIs of FA mothers themselves is significantly lower than those of $\mathrm{N}$ mothers and a diet synchronized with the elimination-substitution diet therapy for children may be one of the factors contributing to the low BMI [10]. In this study, the objective was to understand the meal content and the features of the meals by focusing on the meal content synchronized with the elimination-substitution diet of the child, i.e., the correlation of meal content between the mother and the child and the degree and the dietary patterns of the combined meal content of the mother and child.

Many studies of diet similarities in families have focused on the intake of nutrients and on the intake of specific foods or food groups. Within the family, similarities reportedly vary depending on the age of the parent-child pair or the child-child pair and the role of the parent and similarities in intake of non-core food groups are reportedly highly correlated between fathers and children [23-28]. On the other hand, there have been reports of the similarity between the intake of the mother and child being higher than that between the intake of the father and child [14]. Mothers are reportedly more likely than fathers to be aware of their responsibility for controlling intake and are more likely to intensify the surveillance of their children's food intake when dietary control or dietary therapy is required $[14,29,30]$.

In terms of the correlation of meal content between the mother and child in the present study, the correlation coefficient was higher in the FA group than in the $\mathrm{N}$ group. These results suggest that compared with the $\mathrm{N}$ mother, the FA mother may have a more synchronized diet with the children. However, in the evaluation of dietary patterns of the combined meal content of the mother and child, dietary patterns that summarized the diets of mother and child combined were derived in the $\mathrm{N}$ group, but in the FA group elements for only the mother or the child were derived for the first, second, and third principal components, showing that there were diverging trends. These results suggest that a diet similar to the child's elimination-substitution diet but with some differing characteristics is consumed. In addition, when the intake adequacy by food group was compared, although there were similarities between the mother and child in the intake of allergy-causing foods, such as egg and substitute foods such as meat and fish, there were differences between the mother and the child in the way the substitute foods, such as for potatoes, sugars, and sweets, were consumed. When a comparison between the FA and $\mathrm{N}$ groups was performed, intake adequacy of sweets in FA mothers was significantly higher than that in N mothers. The intake adequacy of sweets was considered to be the point of importance. The cause for this finding was considered to be related to the dietary environment of FA mothers. FA mothers have a background where they are in a dietary environment in which the strict enforcement of diet therapy is essential, and the use of substitute diets is required. Strict enforcement of diet therapy places a considerable burden on the mind and body, even if the number of restricted foods is minimized [31-33]. Mothers must be aware of adherence to diet and enhanced surveillance of the children is added. As such, an increase in stress is considered to be present. In addition, when diet therapy for children becomes a priority, a situation where food for the mothers themselves becomes insufficient may arise and they may supplement their diets with sweets. Even if sweets are consumed, if the meal content is adjusted for the day, there will not be many nutritional problems. However, if the situation lasts more than a day, the dietary habit of eating sweets may lead to an imbalance in diet and nutrition, and as a result, may be involved in the observance of low BMI. When sweets (e.g. rice confectioneries) are consumed, there is a risk for nutritional imbalance because side dishes are not consumed along with the sweets. Because the mothers who responded that they themselves had used substitute food had significantly lower BMI, an imbalance between the burden on the mind and body and diet and nutrition is surmised. In the future, comprehensive evaluations of lifestyle habits, including the dietary habits of FA mothers, are needed and nutritional support for FA mothers may be required.

\section{Study limitations}

This study has several limitations that need to be considered. First, this is a cross-sectional study and causality cannot be proven. Second, the small sample size may limit the interpre- 
tation of the results. Third, multivariate analysis did not consider confounding factors, such as socioeconomic status or the impact of exercise habits. Fourth, the mothers' height and weight values and responses to mother-child dietary surveys were self-reported by mothers, and there may be recall bias. As such, the possibility of some error must be considered.

\section{Conclusions}

In conclusion, in FA children, it was confirmed that the adherence to elimination-substitution diet therapy led to typical growth. However, the BMIs of FA mothers were significantly lower than those of $\mathrm{N}$ mothers. The reason was that although the meal contents of FA mothers were highly similar to the elimination-substitution diet provided to children, there may be some discrepancies. Furthermore, there was a negative relationship between the mother's response to the use of substitute foods and BMI. Based on the aforementioned findings, FA mothers are considered to be in a dietary environment where substitute diets need to be provided, and because the mothers consume meals that slightly differ from the substitute diet for the child, and there is a dietary intake habit of consuming sweets and an imbalance between diet and nutrition, this is thought to have led to the low BMI of FA mothers.

\section{Acknowledgments}

We would like to express our gratitude to the parents who took their children for care at the Pediatrics Department of the Osaka Prefectural Medical Center for Respiratory and Allergic Diseases (currently known as the Osaka Habikino Medical Center) and who kindly consented to participate in this study.

\section{Financial Disclosure}

This work was supported by JSPS KAKENHI, Grant number: JP17K00950.

\section{Conflict of Interest}

None to declare.

\section{Informed Consent}

The study content was explained to the subjects beforehand, and their written consent was obtained. Consent was also obtained for the use of sensitive information in this study, and personal information was pseudonymized when conducting the analysis.

\section{Author Contributions}

Yasuko Fukuda drew up the research plan, dealt with ethical re- search screening, provided detailed explanation of the research to the subjects, collected/analyzed the data, and prepared the initial draft. Makoto Kameda drew up the research plan, dealt with the ethical research screening, explained the research to the subjects, and obtained their consent.

\section{References}

1. Isojima T, Naiki Y, Horikawa R, Yokoya S, Tanaka T. Correlation between body mass index (BMI) z score and $\%$ overweight $(\% \mathrm{OW})$ in Japanese children: a hospitalbased study. J Jpn Ass Hum Auxo. 2007;13(2):69-77.

2. Isojima T, Naiki Y, Horikawa R, Yokoya S, Tanaka T. Correlation between body mass index $\mathrm{z}$ score and percent obesity in Japanese children in Akita prefecture. J Jpn Soc Stu Obes. 2008;14(2):159-165.

3. Fujii K, Ishizaki A, Ogawa A, Asami T, Kwon H, Tanaka A, Sekiya N, et al. Validity of using multi-frequency bioelectrical impedance analysis to measure skeletal muscle mass in preschool children. J Phys Ther Sci. 2017;29(5):863-868.

4. Freedman DS, Horlick M, Berenson GS. A comparison of the Slaughter skinfold-thickness equations and BMI in predicting body fatness and cardiovascular disease risk factor levels in children. Am J Clin Nutr. 2013;98(6):1417-1424.

5. Wohlfahrt-Veje C, Tinggaard J, Winther K, Mouritsen A, Hagen CP, Mieritz MG, de Renzy-Martin KT, et al. Body fat throughout childhood in 2647 healthy Danish children: agreement of BMI, waist circumference, skinfolds with dual X-ray absorptiometry. Eur J Clin Nutr. 2014;68(6):664-670.

6. Sun Q, van Dam RM, Spiegelman D, Heymsfield SB, Willett WC, Hu FB. Comparison of dual-energy x-ray absorptiometric and anthropometric measures of adiposity in relation to adiposity-related biologic factors. Am J Epidemiol. 2010;172(12):1442-1454.

7. Flegal KM, Graubard BI. Estimates of excess deaths associated with body mass index and other anthropometric variables. Am J Clin Nutr. 2009;89(4):1213-1219.

8. Meyer R, De Koker C, Dziubak R, Venter C, DominguezOrtega G, Cutts R, Yerlett N, et al. Malnutrition in children with food allergies in the UK. J Hum Nutr Diet. 2014;27(3):227-235.

9. Chong KW, Wright K, Goh A, Meyer R, Rao R. Growth of children with food allergies in Singapore. Asia Pac Allergy. 2018;8(4):e34.

10. Fukuda Y, Yamamoto S, Nishida K, Takaoka Y, Kameda M. A study on the effect of nutrient intake on the body mass index of mothers of children with food allergies. Asian Pac J Allergy Immunol. 2019.

11. Yoshiike N. Current status of nutrition and diet in Japanese infants and preschool children-measures and future direction. J Natl Inst Public Health. 2017;66(6):566-573. (in Japanese).

12. Agosti M, Tandoi F, Morlacchi L, Bossi A. Nutritional and metabolic programming during the first thousand days of life. Pediatr Med Chir. 2017;39(2):157. 
13. Zuercher JL, Wagstaff DA, Kranz S. Associations of food group and nutrient intake, diet quality, and meal sizes between adults and children in the same household: a cross-sectional analysis of U.S. households. Nutr J. 2011;10:131.

14. Vepsalainen H, Nevalainen J, Fogelholm M, Korkalo L, Roos E, Ray C, Erkkola M, et al. Like parent, like child? Dietary resemblance in families. Int J Behav Nutr Phys Act. 2018;15(1):62.

15. Rosner B, Prineas R, Loggie J, Daniels SR. Percentiles for body mass index in U.S. children 5 to 17 years of age. J Pediatr. 1998;132(2):211-222.

16. Takahashi K, Yoshimura Y, Kaimoto T, Kunii D, Komatsu T, Yamamoto S. Validation of a food frequency questionnaire based on food groups for estimating Individual nutrient intake. Jpn J Nutr. 2001;59(5):221-232. (in Japanese).

17. Kobayashi S, Honda S, Murakami K, Sasaki S, Okubo H, Hirota N, Notsu A, et al. Both comprehensive and brief self-administered diet history questionnaires satisfactorily rank nutrient intakes in Japanese adults. J Epidemiol. 2012;22(2):151-159.

18. Hishida A, Sasaki S. Ministry of Health, Labour and Welfare "Dietary Reference Intake for Japanese, 2015" formulation review committee report. Dietary Reference Intake for Japanese. 2015. Dai-ichi Shuppan. (in Japanese).

19. Mochida K, Toga S, Akamatsu R, Ichi I, Fujiwara Y. Implementing a nutrition education program using the Japanese food guide spinning top and behavioral science. JJHEP. 2016;24(4):231-238. (in Japanese).

20. Kant AK. Dietary patterns and health outcomes. J Am Diet Assoc. 2004;104(4):615-635.

21. Hoffer LJ. Human protein and amino acid requirements. JPEN J Parenter Enteral Nutr. 2016;40(4):460-474.

22. Semba RD, Shardell M, Sakr Ashour FA, Moaddel R, Trehan I, Maleta KM, Ordiz MI, et al. Child stunting is associated with low circulating essential amino acids. EBioMedicine. 2016;6:246-252.

23. Beydoun MA, Wang Y. Parent-child dietary intake resemblance in the United States: evidence from a large representative survey. Soc Sci Med. 2009;68(12):2137-2144.

24. Shrivastava A, Murrin C, Sweeney MR, Heavey P, Kelle- her CC, Lifeways Cohort Study Steering G. Familial intergenerational and maternal aggregation patterns in nutrient intakes in the Lifeways Cross-Generation Cohort Study. Public Health Nutr. 2013;16(8):1476-1486.

25. Hall L, Collins CE, Morgan PJ, Burrows TL, Lubans DR, Callister R. Children's intake of fruit and selected energydense nutrient-poor foods is associated with fathers' intake. J Am Diet Assoc. 2011;111(7):1039-1044.

26. Wroten KC, O'Neil CE, Stuff JE, Liu Y, Nicklas TA. Resemblance of dietary intakes of snacks, sweets, fruit, and vegetables among mother-child dyads from low income families. Appetite. 2012;59(2):316-323.

27. Cooke LJ, Wardle J, Gibson EL, Sapochnik M, Sheiham A, Lawson M. Demographic, familial and trait predictors of fruit and vegetable consumption by pre-school children. Public Health Nutr. 2004;7(2):295-302.

28. Raynor HA, Van Walleghen EL, Osterholt KM, Hart CN, Jelalian E, Wing RR, Goldfield GS. The relationship between child and parent food hedonics and parent and child food group intake in children with overweight/obesity. J Am Diet Assoc. 2011;111(3):425-430.

29. Oliveria SA, Ellison RC, Moore LL, Gillman MW, Garrahie EJ, Singer MR. Parent-child relationships in nutrient intake: the Framingham Children's Study. Am J Clin Nutr. 1992;56(3):593-598.

30. Wang Y, Beydoun MA, Li J, Liu Y, Moreno LA. Do children and their parents eat a similar diet? Resemblance in child and parental dietary intake: systematic review and meta-analysis. J Epidemiol Community Health. 2011;65(2):177-189.

31. Hu W, Grbich C, Kemp A. Parental food allergy information needs: a qualitative study. Arch Dis Child. 2007;92(9):771-775.

32. Xu YS, Waserman SB, Waserman S, Connors L, Stawiarski K, Kastner M. Food allergy management from the perspective of patients or caregivers, and allergists: a qualitative study. Allergy Asthma Clin Immunol. 2010;6(1):30.

33. Vargas PA, Sicherer SH, Christie L, Keaveny M, Noone S, Watkins D, Carlisle SK, et al. Developing a food allergy curriculum for parents. Pediatr Allergy Immunol. 2011;22(6):575-582. 